\title{
Seasonal Onset of Insulin Dependent Diabetes in Relation to Sex and Age at Onset
}

\author{
R. S. Gray, L. J. P. Duncan, and B. F. Clarke \\ Diabetic and Dietetic Department, Royal Infirmary, Edinburgh, Scotland
}

\begin{abstract}
Summary. All newly diagnosed insulin dependent diabetics presenting consecutively to the Diabetic Department of the Royal Infirmary from the City of Edinburgh or its environs, between the years 1964 and 1977, were analysed for sex (297 males, 205 females), age at diagnosis (range 10-75 years), month of diagnosis, duration of symptoms and month of symptomatic onset. Males aged 10-19 years showed a significant seasonal variation in diagnosis $(\mathrm{p}<0.025)$ with an increase in autumn and winter months which was not seen in females aged 10-19 years nor in patients of either sex aged more than 19 years at diagnosis. The duration of diabetic symptoms increased with increasing age at diagnosis in both males and females and was consistantly greater in females than in males for each age group at diagnosis. When the month of symptomatic onset was considered in relation to sex and age at diagnosis, having excluded patients with duration of symptoms of more than three months, only males aged 10-19 years at diagnosis showed a significant seasonal variation in incidence $(\mathrm{p}<0.005)$.
\end{abstract}

Key words: Seasonal incidence, insulin dependent diabetes, age at onset, duration of symptoms, sex.

In 1926, Adams demonstrated "a seasonal variation in the onset of acute diabetes" irrespective of sex and age at onset [1]. Gamble et al. [2] have since reported a seasonal variation in the incidence of insulin dependent diabetes in patients presenting under the age of 20 years and this phenomenon has been confirmed in further studies $[3,4,5]$, of juvenile onset insulin dependent diabetics (IDD). Where an attempt has been made to show a seasonal variation in the inci- dence of newly diagnosed insulin dependent diabetes presenting over the age of 20 years, the evidence is less convincing [2], although the decline in incidence during the early summer months remains evident when all patients presenting below the age of 30 years are considered together [3], or when all patients are considered irrespective of age at onset [1]. Analysis of patients presenting below the age of 6 years has revealed a lack of the seasonal variation in incidence which occurs in patients presenting between 6 and 20 years $[4,5]$.

No attempt has been hitherto made to compare the patterns of seasonal incidence of insulin dependent diabetes presenting in males and females, nor to consider that of patients presenting over the age of 30 years. If the seasonal variation in incidence provides a possible clue to the aetiology of insulin dependent diabetes, it is necessary to establish which patients exhibit this phenomenon. We have therefore examined the effects of sex and age of onset on the pattern of seasonal incidence of insulin dependent diabetes as observed in one large adult diabetic clinic.

\section{Patients and Methods}

All patients with newly diagnosed diabetes who had been referred to the Diabetic Department of the Royal Infirmary, Edinburgh from the city or its environs, between 1964 and 1977 (inclusive) were considered. Patients were regarded as insulin dependent according to their age at presentation, if underweight and/or ketonuric and only those treated with insulin from diagnosis and for at least six months thereafter were included. Patients with secondary diabetes, due to pancreatic disease, endocrine disturbance or other causes were excluded. The study did not include patients aged less than 10 years and many others aged 10-13 years who had been referred initially to a paediatric clinic. The majority (ie more than $80 \%$ ) of diabetics in Edinburgh attend the Diabetic Department at the Royal Infirmary [6] and will have therefore been included in our study. 


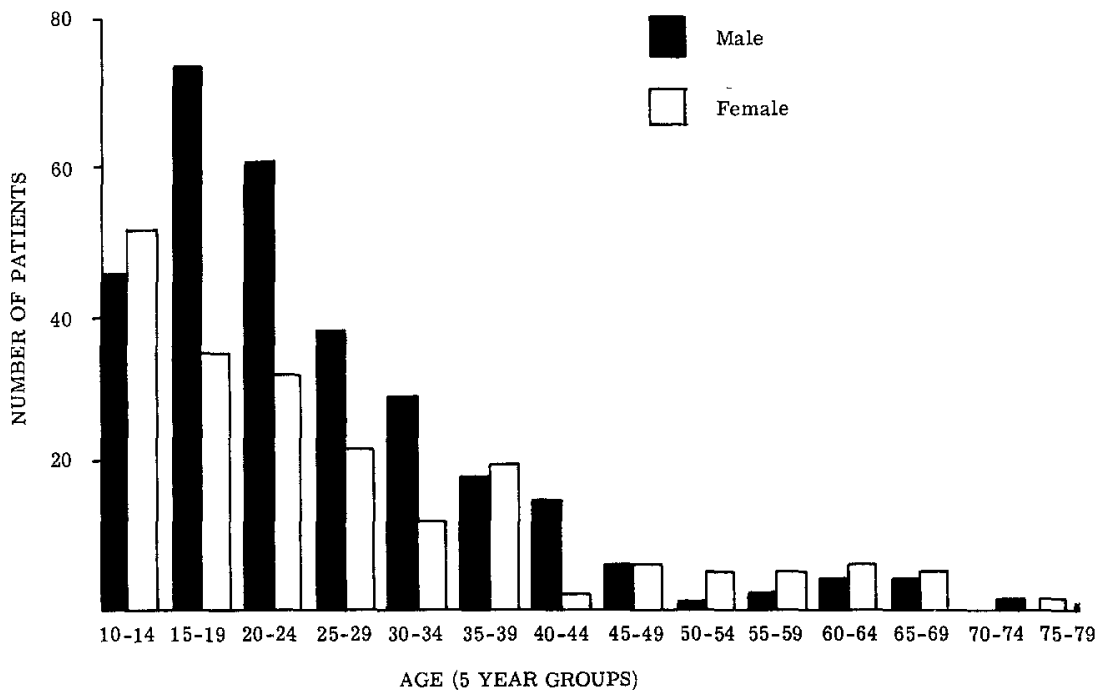

Fig. 1. Age and sex distribution of patients studied

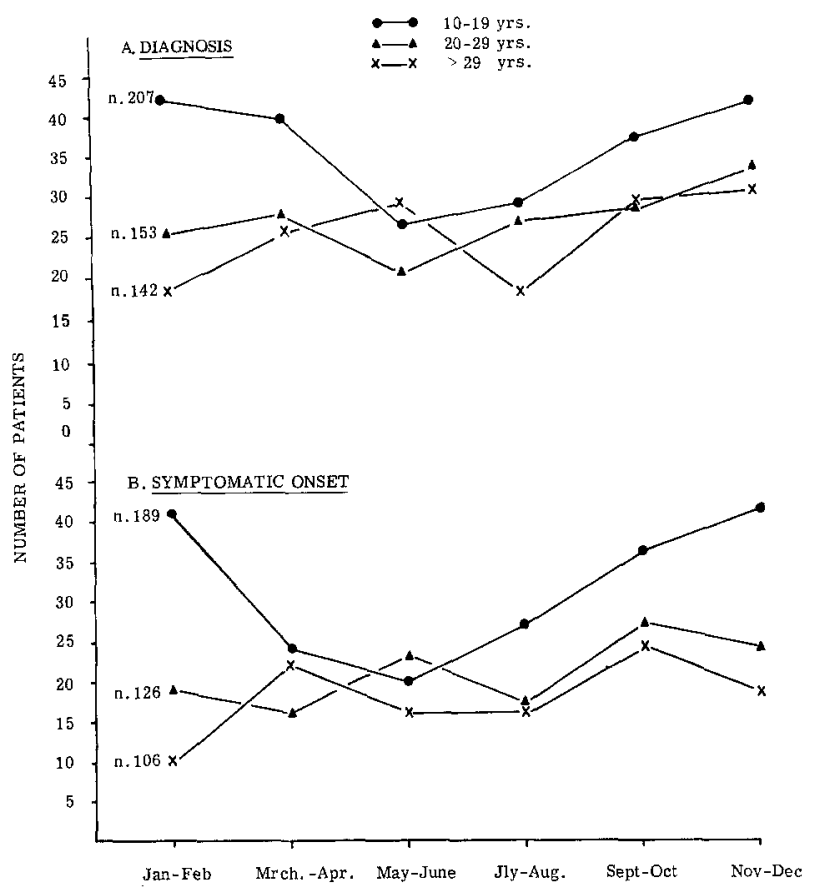

Fig. 2. Pattern of seasonal incidence of diagnosis (A), and of symptomatic onset (B), according to age at onset

Five hundred and two patients (297 males, 205 females) were included and analysed for the year and month of diagnosis and of symptomatic onset. The duration of diabetic symptoms before diagnosis had been obtained from all but 9 patients at diagnosis. For the purpose of analysis, patients were sub-divided into three groups according to age at onset $(10-19,20-29$ and more than 29 years).

Statistical analysis of the seasonal variation in incidence was made by Rayleigh's test [7] (parametric) and a test due to David \& Newell [8] (non-parametric). The duration of symptoms of IDD of differing sex and age at onset were compared using Cox's test for trend [9].

\section{Results}

The age at diagnosis and sex distribution of the 502 IDD studied are shown in Figure 1. The mean annual incidence of diabetes was 14.8 (range 8-22) for patients aged 10-19, 10.9 (range 5-19) for those aged 20-29 and 10.0 (range 6-15) for those aged more than 29 years.

Figure $2 \mathrm{~A}$ compares the seasonal variation in incidence of diagnosis of diabetes of the three age groups in patients of both sexes combined. In patients aged 10-19 years at diagnosis, the incidence of diagnosis was significantly greater during the winter months than during the summer months $(\mathrm{p} \leqslant 0.05)$ by parametric and non-parametric analysis. Patients aged 20-29 and more than 29 years failed to show a significant seasonal variation in the incidence of diagnosis. When males and females aged 10-19 and more than 19 years (combining the patients aged 20-29 and more than 29 years at diagnosis) were considered separately (Table 1 ), only the males aged $10-19$ years showed a significant seasonal variation in incidence of diagnosis $(\mathrm{p}<0.025$, Rayleigh's test and $<0.05$, David \& Newell's test).

Figure 3 compares the duration of diabetic symptoms experienced by IDD of different sex and age groups. When the sexes were considered together, the duration of symptoms of IDD aged 10-19 years was significantly shorter than that of IDD aged 20-29 years $(p<0.02)$ and IDD aged more than 29 years $(p<0.0001)$. Women within each age group had a longer duration of symptoms than men but the difference was only statistically significant $(p<0.05)$ in patients aged 20-29 years at diagnosis.

Figure $2 \mathrm{~B}$ shows the seasonal variation in symptomatic onset of diabetes in 421 patients according to 
Table 1. Diagnosis and symptomatic onset of male and female insulin dependent diabetics according to age at onset and time of year

\begin{tabular}{|c|c|c|c|c|c|c|c|c|}
\hline & Age (yrs) & Sex & Jan.-Feb. & March-April & May-June & July-Aug. & Sept.-Oct. & Nov.-Dec. \\
\hline \multirow[t]{4}{*}{ Diagnosis } & \multirow[t]{2}{*}{$10-19$} & M & 21 & 22 & 13 & 11 & 22 & 29 \\
\hline & & $\mathbf{F}$ & 21 & 17 & 12 & 16 & 13 & 10 \\
\hline & \multirow[t]{2}{*}{$>19$} & M & 25 & 33 & 32 & 27 & 29 & 33 \\
\hline & & $\mathrm{F}$ & 18 & 19 & 15 & 14 & 24 & 26 \\
\hline \multirow[t]{4}{*}{ Symptomatic onset } & \multirow[t]{2}{*}{$10-19$} & $\mathbf{M}$ & 20 & 15 & 8 & 16 & 22 & 27 \\
\hline & & $\mathrm{F}$ & 21 & 9 & 12 & 11 & 14 & 14 \\
\hline & \multirow[t]{2}{*}{$>19$} & M & 18 & 25 & 25 & 23 & 30 & 28 \\
\hline & & $F$ & 9 & 15 & 13 & 11 & 21 & 14 \\
\hline
\end{tabular}

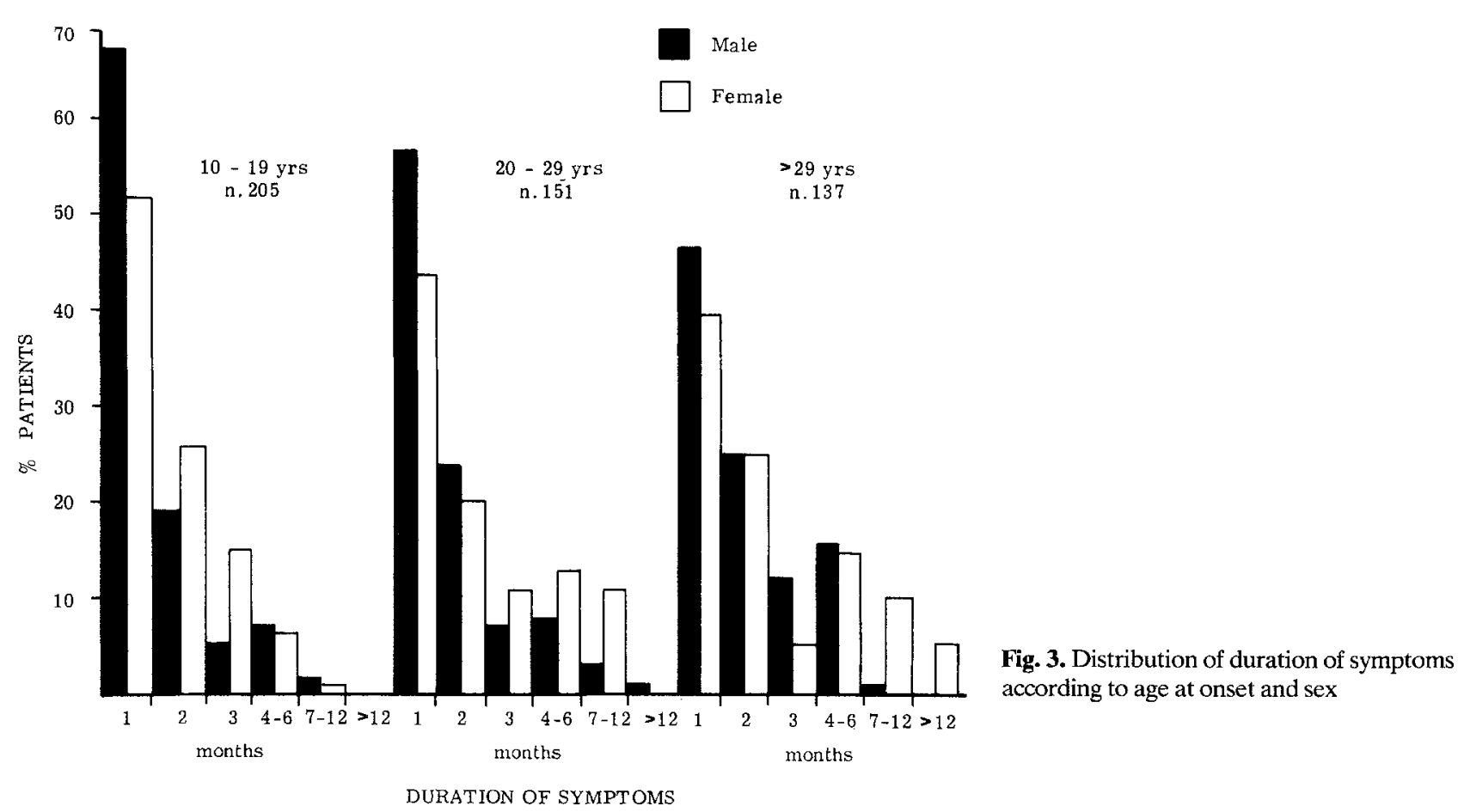

age at diagnosis, having excluded 72 patients whose symptoms had been present for more than three months prior to diagnosis and 9 patients whose duration of symptoms was unknown. In patients of both sexes combined aged 10-19 years at diagnosis, the incidence of symptomatic onset was again commoner during the winter months than during the summer months ( $\mathrm{p}<0.005$, Rayleigh's test and $<0.01$, David $\&$ Newell's test). Patients of both sexes combined aged 20-29 years and more than 29 years at diagnosis failed to show a seasonal variation in symptomatic onset. When males and females aged 10-19 and more than 19 years (combining the patients age 20-29 and more than 29 years at diagnosis) were considered separately (Table 1), only the males aged 10-19 years showed a significant seasonal variation in incidence of symptomatic onset $(p<0.005$ Rayleigh's test and $<0.05$, David \& Newell's test).

\section{Discussion}

The distribution of age and sex at presentation of our patients accords with previous reports, $[2,10]$ having considered the referral of some younger teenagers and patients aged less than 10 years to paediatric clinics. As with other recent studies, $[2,3,4,5]$ we have observed a seasonal variation in incidence of insulin dependent diabetes presenting between the ages of 10-19 years - the incidence being highest between the months of September and April inclusive. However, of patients aged $10-19$ years at diagnosis, only males showed a significant seasonal variation in incidence. It is of interest that patients aged over 19 years failed to show such a pattern of seasonal incidence, whether considering the sexes separately or combined.

A significant seasonal variation in incidence of 
insulin dependent diabetes might only become evident when the duration of symptoms prior to diagnosis is taken into account and those patients having a particularly lengthy symptomatic onset excluded. Males aged 10-19 years at diagnosis showed a very marked seasonal variation in symptomatic onset. In contrast, females aged 10-19 and patients of each sex aged more than 19 years at diagnosis, showed no seasonal variation in incidence even when their duration of symptoms was taken into account, although it is evident that the duration of symptoms prior to diagnosis increases with increasing age at onset of diabetes and is consistantly longer in female than in male IDD. Such a prolonged interval between the onset and diagnosis of diabetes may obscure the seasonal variation in incidence as shown by the younger male patients, whose duration of symptoms is relatively short.

An alternative interpretation of our findings is that there may be a true aetiological distinction between juvenile and late-onset IDD and possibly between female and male IDD. Reports of HLA antigen frequencies in diabetic populations show insulin dependent diabetes to be a heterogenous disorder within which the prevalence of some HLA antigens may vary with age at onset. Thus, the prevalence of HLA, A1, A11, B8, B 18 and B15 may not be as high in late onset as in juvenile IDD [11]. This observation is of particular interest in light of the suggested clustering of HLA identical diabetic siblings [12] and HLA B 8/B 15 positive phenotypes during the winter peak [10] and increased frequency with which high antibody titres to Coxsackie B 1-4 may be found in HLA B 8/B 15 positive patients [10]. Thus, it could be postulated that late onset IDD include fewer patients liable to develop diabetes in the winter months by virtue of their HLA status and for this reason fail to exhibit the seasonal variation in onset found in younger patients.

The development of type I diabetes may derive from the interaction between immunological responsiveness and an environmental factor or factors. Ageing, per se, influences immunological responsiveness and appears to increase the risk of the development of organ-specific auto-immune disease. Certainly, the clinical association between type I diabetes and organ-specific auto-immune disease is predominantly found in female late-onset IDD [13]. A high propor- tion of such late onset IDD may prove to have an autoimmune aetiology. By contrast, the younger male IDD show a variable seasonal incidence which may reflect a possible infective aetiology.

Acknowledgements. The authors gratefully acknowledge the invaluable assistance provided by Sarah Dickson, Lynne Forrest and Elizabeth Wilson.

\section{References}

1. Adams, S. F.: The seasonal variation in the onset of acute diabetes. Arch. Intern. Med. 37, 861-864 (1926)

2. Gamble, D. R., Taylor, K. W.: Seasonal incidence of diabetes mellitus. Br. Med. J. 1969 II, 631-633

3. Christau, B., Kromann, H., Andersan, O. O., Christy, M., Buschard, K., Arnung, K., Kristensen, I. H., Peitersen, B., Steinrud, J., Nerup, J.: Incidence, seasonal and geographical patterns of juvenile-onset insulin-dependent diabetes mellitus in Denmark. Diabetologia 13, 281-284 (1977)

4. Bloom, A., Hayes, T.M., Gamble, D. R.: Register of newly diagnosed diabetic children. Br. Med. J. 1975 III, 580-583

5. McMillan, D. R., Kotoyan, M., Zeidner, D., Hafezi, B.: Seasonal variation in the onset of diabetes in children. Pediatrics 59, 113-115 (1977)

6. Falconer, D. S., Duncan, L. J. P., Smith, C.: A statistical and genetic study of diabetes 1 . Prevalence and morbidity. Ann. Hum. Genet. 34, 347-369 (1971)

7. Mardia, K. V.: Statistics of directional data, p. 133-135. New York: Academic Press 1972

8. David, H. A., Newell, D. J.: The identification of annual peak periods for a disease. Biometrics 21, 645-650 (1965)

9. Cox, D. R.: Analysis of binary data, p. 65-66. London: Methven \& Co. Ltd. 1970

10. Cudworth, A. G., Gamble, D. R., White, G. B. B., Lendrum, R., Woodrow, J. C., Bloom, A.: Aetiology of juvenile onset diabetes. Lancet 1977 I, 385-388

11. Cudworth, A.G., Woodrow, J.C.: Genetic susceptibility in diabetes mellitus: Analysis of the HLA association. Br. Med. J. 1976 II, 846-848

12. Barbosa, J., King, R., Noreen, H., Yunis, E. J.: The histocompatability system in juvenile, insulin-dependent diabetic multiplex kindreds. J. Clin. Invest. 60, 989-998 (1977)

13. Bottazzo, G. F., Cudworth, A. G., Moul, D. J., Doniach, D., Festenstein, H.: Evidence for a primary autoimmune type of diabetes mellitus. Br. Med. J. 1978 I, 1253-1255

Received: November 28, 1978

and in revised form: April 6, 1979

Dr. R. S. Gray

Diabetic and Dietetic Department

Royal Infirmary

Edinburgh, EH3 9YW

Scotland 